\title{
Influence of Intense Swimming Exercise on Selected Salivary Components
}

\author{
Iwona Grzesiak-Gasek ${ }^{1 *}$, Dorota Diakowska ${ }^{2}$, Urszula Kaczmarek ${ }^{1}$
}

\author{
${ }^{1}$ Department of Conservative Dentistry and Paediatric Dentistry, Wroclaw Medical University, Rektorat, wybrzeże Ludwika Pasteura 1, 50-367 \\ Wrocław, Poland \\ ${ }^{2}$ Department of Nervous System Disease Faculty of Health Science, Wroclaw Medical University, Rektorat, wybrzeże Ludwika Pasteura 1, 50-367 \\ Wrocław, Poland
}

DOI: $10.36347 /$ sjds.2020.v07i01.002

| Received: 30.12.2019 | Accepted: 06.01.2020 | Published: 14.01.2020

*Corresponding author: Iwona Grzesiak-Gasek

Abstract

Original Research Article

Physical exercise may induce many changes in quantity and quality of the biochemical components of saliva. Therefore, a noninvasive sampling and testing of saliva can be used as a diagnostic method in monitoring of physical activity. Objectives of this study was comparison selected salivary components in swimmers before and after intense training. 19 subjects of both sexes, 18-24 years of age, were selected for the study, of which 10 were competitive swimmers and 9 were in the control group. Salivary samples were collected from all subjects in the morning and in the afternoon and from the swimmers before and after training. The parameters measured included: salivary flow rate, $\mathrm{pH}$, total protein, alpha-amylase, peroxidase, cortisol, total antioxidant status (TAS), sialic acid (FSA, GSA, TSA), calcium and magnesium. A significant increase of salivary, alpha-amylase and GSA was noted in all swimmers after the morning workout, while after the afternoon swim training significant decrease in the salivary flow rate and cortisol, accompanied by increasing protein and alpha-amylase levels, were found. Regarding the output of salivary components, after the morning training session, a slight increase in the output was observed for all salivary parameters being investigated. However, it was statistically significant only for total protein, alpha-amylase, calcium, TSA and TAS. After the afternoon training a slight increase in the output was observed for all investigated parameters, except for cortisol which showed decreasing trend. A statistically significant increase in the output was recorded for total protein, alpha-amylase, calcium and TAS. The data suggest a significant increase in alpha-amylase levels and tendency for greater total protein concentration in saliva, following morning and afternoon swimming workout.

Keywords: Physical exercises, Swimmers, Saliva, Physiological, Biomarkers.

Copyright @ 2019: This is an open-access article distributed under the terms of the Creative Commons Attribution license which permits unrestricted use, distribution, and reproduction in any medium for non-commercial use (NonCommercial, or CC-BY-NC) provided the original author and source are credited.

\section{INTRODUCTION}

Physical activity induces changes in biochemical components of blood, which are reflected in saliva. Therefore, saliva constitutes an alternative diagnostic material, which can be used for monitoring of the body's response to physical activity and to psychological stress related to playing sports, especially that saliva can be collected in an easy, non-invasive manner. Both the salivary flow rate and its composition are dependent on the autonomic nervous system's activity and every change in this activity can cause changes in the composition of saliva. During physical activity a sympathetic system stimulation can occur and it is, strong enough to diminish or significantly reduce salivary secretion $[1,2]$. When considering physical activity, its type, course, intensity, duration and the time of day need to be taken into account. However, studies have showed a differential impact of physical activity on salivary secretion, ranging from no influence [3] to significant increase [4] or decrease [5, 6]. That can be caused, to some extent, by the time of day, because the sympathetic nervous system's activity is higher in the morning than at other times of the day [5]. Decrease in salivary secretion during intense exercises is explained by an increase in sympathetic nervous system's activity leading to the narrowing of blood vessels which deliver blood to salivary glands and consequently, to reduce salivary volume. The parasympathetic system's activity is known to promote its secretion [6]. Moreover, the physical activity-induced increase in vasopressin (antidiuretic hormone) levels can decrease saliva secretion. The influence of dehydration during exercise, evaporation of saliva due to breathing through the mouth as well as hyperventilation, cannot be ignored, either [7].

Physical exercises can also cause some changes in the concentration of organic and inorganic components of saliva. Salivary proteins are released 
mainly after activation of the sympathetic nervous system. Moreover, the level of cortisol in the blood (glucocorticoid hormone produced by the adrenocortical glands, called the stress hormone) increases after physical exercise, which largely influences the body's metabolic rate. The unbound protein fraction of cortisol travels by passive diffusion to body fluids, including saliva. Since a correlation has been found between cortisol levels in blood serum and in saliva, salivary cortisol can be an indicator of the adrenocortical gland's secretions during physical activity [8]. Circadian rhythm of the blood cortisol was identified to reach its maximum level early in the morning, decreasing further into the day to reach the low level in the evening and the cortisol concentration was found to increase following physical exercise performed in the morning rather than in the evening [9]. Also, the concentration of alpha-amylase, a salivary enzyme, which constitutes half of total salivary protein, was shown to increase in response to psychological and physical stress [6]. Alpha-amylase, produced mainly in parotid and submandibular salivary glands, is a sensitive marker reflecting sympathetic nervous system activity and, constitutes an alternative to both catecholamines (adrenaline and noradrenaline) and cortisol assays [10].

Assessment of lactate concentration in blood serum can be also used for monitoring athletes' training. Lactate provides an anaerobic threshold based on the blood lactate levels measured at the lower limits of training intensity. In saliva, just like in the blood serum, a significant increase in the lactate concentration was observed and it correlated with its levels in the blood serum and saliva, after the swim training [11]. A study of Immunoglobulin A, the first line of defense against upper respiratory tracts' infections in response to physical activity, is not completely unambiguous. Since both the body's metabolic rate and oxygen consumption increase during physical activity (due to increased metabolic rate especially in skeletal muscles), the production of reactive oxygen species (ROS) increases, as well. Under the normal conditions, reactive oxygen species are eliminated by intracellular and extracellular defense mechanisms, including enzymatic and non-enzymatic antioxidants which delay or inhibit oxidation of substrates [12]. An increase in the antioxidant levels in the saliva was observed after physical activity [13]. Levels of trace elements and electrolytes have been assessed, with magnesium, sodium, and manganese contents increasing in saliva after physical activity [14].

The aim of the study was to evaluate selected salivary components and their circadian rhythm in swimmers before and after swim training and compare them with sedentary controls.

\section{MATERIALS AND METHODS}

Participants: 19 subjects aged 18-24 (mean 23) were included in the study. The Study group consisted of 10 competitive swimmers: 7 males, and 3 females. The Control group consisted of 9 subjects with sedentary lifestyles: 4 males and 5 females. Inclusion criteria were as follows: subjects at the age of 18 and over, generally healthy, not taking any medicine, who practice swimming for 20 hours a week, or who are currently not engaged in any physical activity.

All subjects were provided with a detailed description of the study protocol and then a written informed consent was obtained from them. The swimmers trained 21 hours per week in the morning (7.00-8.45 a.m.) and in the afternoon (4.00-5.45 p.m.). The swim training consisted of swimming the distance of 5000 meters.

\section{Salivary sample collection and analysis}

To ensure consistency and to account for diurnal fluctuation in the salivary parameters, all unstimulated mixed saliva samples were taken from swimmers in the morning and in the afternoon, before and after the swimming workout. In the study group, saliva samples were collected 4 times a day (i.e. in the morning and in the afternoon before and after swim training). The total number of saliva samples collected amounted to 40 . In the control group, saliva was collected twice a day, in the morning and in the afternoon, at the time when the study group started the swim training. The total number of saliva samples collected from the control group amounted to 18 .

Samples of saliva were collected after a thorough mouth rinse with distilled water. The subjects were sitting, head bent down and the mouth open. Saliva was taken with a plastic pipette and put into a graded test tube placed on crushed ice. Based on the measurement of the volume of the collected saliva sample, and the time needed to collect it, the salivary flow rate was calculated as $\mathrm{ml} / \mathrm{min}(\mathrm{V})$. The samples were centrifuged for 10 minutes at a speed of $3500 \mathrm{rpm}$ before biochemical assays. Sample collection was preceded by rinsing the mouth and clear supernatants were used for the assays to minimize the contribution of other sources of the tested salivary parameters, such as: oral bacteria, epithelial cells, leukocytes, and dietary residues. Total protein - P (by Lowry's et al., method) [11], pH (by potentiometric method), alpha-amylase Amy (by Caraway's colorimetric method-Alpha Diagnostic Kit), salivary peroxidase - SPO (using NbsSCN method) [15], salic acid total - TSA, bound - GSA and free - FSA (by periodate-resorcinol method) [16], cortisol - C (Elisa Kit from R\&D Systems), total antioxidant status - TAS (TAS assay Randox), calcium - Ca (by method based on formation of chromogenic complex between calcium ions and o-cresolphthaelin using Alpha Diagnostics kit), magnesium - Mg (by colorimetric method based on the reaction of 
magnesium with Xylidyl Blue-I using Alpha Diagnostics kit) were assessed. Moreover, the data obtained from the measurements were expressed as an output, i.e. concentration of a specific component obtained within 1 minute.

This study protocol has been approved by The Bioethics Committee (No KB-327/2009) of The Medical University of Wroclaw. All research was performed in accordance with relevant guidelines and regulations and a statement confirming that informed consent was obtained from all participants.

Statistical analysis: Depending on the type of variables distribution, Student's t-test or Mann-Whitney test was used for the independent variables analysis. For the dependent variables, Student's t-test or nonparametric Wilcoxon's test was conducted. The obtained data were analyzed statistically with the significance level set at $\mathrm{p}<0.05$ by STATISTICA 13.1 software package (StatSoft, Inc., Tulsa, OK, USA).

\section{RESULTS AND DISCUSSION}

\section{Analysis of the Time of Day Variations}

We found slight fluctuations in the average levels of salivary parameters related to the time of day. This was found in The samples collected both in swimmers before swim training and in the control group. Those levels were slightly lower in saliva collected in the morning hours than in the samples collected in the afternoon for both groups, except for $\mathrm{pH}$, cortisol, and GSA in the control group.

In the control group, the alpha-amylase level was significantly lower in the morning hours compared to the afternoon hours, but it increased in swimmers before the afternoon training session by $41 \%$ ( $p>0.05)$ compared to the morning workout. However, cortisol level was significantly higher in swimmers before the morning training session compared to the afternoon session, with a similar tendency observed in the control group.

Levels of salivary parameters in swimmers before training compared with the control group

In the morning, significantly lower cortisol levels were found in swimmers' saliva compared to the control group. In the afternoon, lower salivary flow rate, higher total protein concentration, lower alphaamylase activity and lower cortisol content were also noted.

Levels of salivary parameters before and after training:

After the morning training session, a significant increase in the levels of total protein, alphaamylase and GSA was observed. Following an afternoon workout, a significant decrease in the salivary flow rate and cortisol concentration, accompanied by increased total protein and alpha-amylase, were observed (Table-1).

Table-1: Concentration of the test components in saliva

\begin{tabular}{|c|c|c|c|c|c|c|}
\hline \multirow[t]{2}{*}{ Salivary parameters } & \multirow{2}{*}{$\begin{array}{l}\text { Day } \\
\text { time }\end{array}$} & \multirow{2}{*}{$\begin{array}{l}\text { Non- swimmers } \\
\text { (Control group) }\end{array}$} & \multicolumn{2}{|c|}{ Swimmers study group } & \multirow{2}{*}{$\begin{array}{l}\text { Before training } \\
\text { swimmers vs non- } \\
\text { swimmers }\end{array}$} & \multirow{2}{*}{$\begin{array}{l}\text { Swimmers before } \\
\text { vs after training }\end{array}$} \\
\hline & & & before & After & & \\
\hline \multirow[t]{2}{*}{$\begin{array}{l}\text { Salivary flow rate }(\mathrm{V}) \\
\mathrm{ml} / \mathrm{min}\end{array}$} & $\begin{array}{l}\mathrm{am} \\
\mathrm{pm}\end{array}$ & $\begin{array}{l}0.53 \pm 0.43 \\
0.60 \pm 0.21\end{array}$ & $\begin{array}{l}0.28 \pm 0.12 \\
0.37 \pm 0.15\end{array}$ & $\begin{array}{l}0.20 \pm 0.87 \\
0.33 \pm 0.19\end{array}$ & $\begin{array}{l}\mathrm{u}=-1.837 \mathrm{p}=0.066^{\mathrm{b}} \\
(\downarrow 47 \%) \\
\mathbf{u}=\mathbf{- 2 . 2 4 5} \mathbf{p}=\mathbf{0 . 0 2 5}^{\mathbf{b}} \\
(\downarrow \mathbf{3 8 \%})\end{array}$ & $\begin{array}{l}\mathrm{t}=3.221 \quad \mathrm{p}=0.089^{\mathrm{a}} \\
(\downarrow 29 \%) \\
\mathrm{t}=0.312 \quad \mathrm{p}=0.583^{\mathrm{a}} \\
(\downarrow 11 \%)\end{array}$ \\
\hline & $\begin{array}{l}\text { am vs } \\
\text { pm }\end{array}$ & $\begin{array}{l}\mathrm{z}=0.652 \mathrm{p}=0.514^{\mathrm{c}} \\
(\uparrow 13 \%)\end{array}$ & $\begin{array}{l}\mathrm{t}=2.156 \\
\mathrm{p}=0.159^{\mathrm{c}} \\
(\uparrow 32 \%)\end{array}$ & $\begin{array}{l}\mathrm{t}=3.894 \\
\mathrm{p}=0.064^{\mathrm{c}} \\
(\uparrow 65 \%)\end{array}$ & & \\
\hline \multirow[t]{2}{*}{$\mathrm{pH}$} & $\begin{array}{l}\mathrm{am} \\
\mathrm{pm}\end{array}$ & $\begin{array}{l}7.48 \pm 0.36 \\
7.33 \pm 0.35\end{array}$ & $\begin{array}{l}6.99 \pm 0.40 \\
7.06 \pm 0.55\end{array}$ & $\begin{array}{l}6.95 \pm 0.41 \\
7.31 \pm 0.19\end{array}$ & $\begin{array}{l}\mathrm{t}=-2.756 \mathrm{p}=0.013^{\mathrm{a}} \\
(\downarrow 6 \%) \\
\mathrm{t}=-1.229 \mathrm{p}=0.235^{\mathrm{a}} \\
(\downarrow 4 \%)\end{array}$ & $\begin{array}{l}\mathrm{t}=0.034 \mathrm{p}=0.855^{\mathrm{a}} \\
(\downarrow 0.57 \%) \\
\mathrm{t}=1.746 \mathrm{p}=0.202^{\mathrm{a}} \\
(\uparrow 3.5 \%)\end{array}$ \\
\hline & $\begin{array}{l}\text { am vs } \\
\text { pm }\end{array}$ & $\begin{array}{l}t=0.799 p=0.384^{\mathrm{a}} \\
(\downarrow 2 \%)\end{array}$ & $\begin{array}{l}\mathrm{t}=0.114 \\
\mathrm{p}=0.739^{\mathrm{a}} \\
(\uparrow 1 \%)\end{array}$ & $\begin{array}{l}\mathrm{t}=5.891 \\
\mathrm{p}=0.025^{\mathrm{a}} \\
(\uparrow 5 \%)\end{array}$ & & \\
\hline \multirow[t]{2}{*}{ Total protein $(\mathrm{P}) \quad \mathrm{g} / \mathrm{l}$} & $\begin{array}{l}\mathrm{am} \\
\mathrm{pm}\end{array}$ & $\begin{array}{l}0.74 \pm 0.21 \\
0.79 \pm 0.22\end{array}$ & $\begin{array}{l}0.79 \pm 0.25 \\
1.26 \pm 0.56\end{array}$ & $\begin{array}{l}1.25 \pm 0.97 \\
1.60 \pm 1.15\end{array}$ & $\begin{array}{l}\mathrm{u}=0.449 \mathrm{p}=0.650^{\mathrm{b}} \\
(\uparrow 7 \%) \\
\mathbf{u}=\mathbf{1 . 9 5 9} \mathbf{p}=\mathbf{0 . 0 5 0} 0^{\mathrm{b}} \\
(\uparrow \mathbf{5 9 \% )}\end{array}$ & $\begin{array}{l}\mathrm{z}=\mathbf{2 . 8 0 3} \mathbf{p}=\mathbf{0 . 0 0 5}^{\mathrm{c}} \\
(\uparrow \mathbf{5 8 \%}) \\
\mathrm{z}=1.718 \mathrm{p}=0.086^{\mathrm{c}} \\
(\uparrow 27.0 \%)\end{array}$ \\
\hline & $\begin{array}{l}\text { am vs } \\
\text { pm }\end{array}$ & $\begin{array}{l}\mathrm{t}=0.157 \quad \mathrm{p}=0.696^{\mathrm{a}} \\
(\uparrow 7 \%)\end{array}$ & $\begin{array}{l}\mathrm{z}=1.783 \\
\mathrm{p}=0.074^{\mathrm{c}} \\
(\uparrow 59 \%)\end{array}$ & $\begin{array}{l}\mathrm{z}=1.579 \\
\mathrm{p}=0.014^{\mathrm{c}} \\
(\uparrow 28 \%)\end{array}$ & & \\
\hline \multirow[t]{2}{*}{$\begin{array}{l}\alpha \text {-amylase (Amy) } \\
\mathrm{j} / \mathrm{ml}\end{array}$} & $\begin{array}{l}\mathrm{am} \\
\mathrm{pm}\end{array}$ & $\begin{array}{l}39.15 \pm 31.30 \\
143.94 \pm 86.55\end{array}$ & $\begin{array}{l}34.48 \pm 19.19 \\
48.50 \pm 33.98\end{array}$ & $\begin{array}{l}50.75 \pm 37.36 \\
62.02 \pm 35.99\end{array}$ & $\begin{array}{l}\mathrm{u}=-0.040 \mathrm{p}=0.967^{\mathrm{b}} \\
(\downarrow 12 \%) \\
\mathrm{t}=\mathbf{- 2 . 8 7 7} \mathrm{p}=\mathbf{0 . 0 0 5} \\
(\downarrow \mathbf{9 5 \%})\end{array}$ & $\begin{array}{l}\mathrm{z}=2.803 \mathrm{p}=0.0005^{\mathrm{c}} \\
(\uparrow 47 \%) \\
\mathrm{z}=1.885 \mathrm{p}=0.059^{\mathrm{c}} \\
(\uparrow 28 \%)\end{array}$ \\
\hline & $\begin{array}{l}\text { am vs } \\
\text { pm }\end{array}$ & $\begin{array}{l}t=11.665 p=0.003^{a} \\
(\uparrow 268 \%)\end{array}$ & $\begin{array}{l}\mathrm{z}=0.764 \\
\mathrm{p}=0.444^{\mathrm{c}} \\
(\uparrow 41 \%)\end{array}$ & $\begin{array}{l}\mathrm{z}=0.561 \\
\mathrm{p}=0.575^{\mathrm{c}} \\
(\uparrow 22 \%)\end{array}$ & & \\
\hline (C) $2019 \mathrm{~S}$ & ent & ences | Published b & AS Publishe & dia & & 6 \\
\hline
\end{tabular}


Iwona Grzesiak-Gasek et al., Sch J Dent Sci, Jan, 2020; 7(1): 4-11

\begin{tabular}{|c|c|c|c|c|c|c|}
\hline \multirow[t]{2}{*}{$\begin{array}{l}\text { Salivary peroxidase } \\
\text { (SPO) } \\
\mathrm{U} / \mathrm{ml}\end{array}$} & $\begin{array}{l}\mathrm{am} \\
\mathrm{pm}\end{array}$ & $\begin{array}{l}1.35 \pm 0.87 \\
1.43 \pm 1.07\end{array}$ & $\begin{array}{l}1.24 \pm 0.65 \\
1.33 \pm 0.89\end{array}$ & $\begin{array}{l}2.05 \pm 2.94 \\
1.39 \pm 0.79\end{array}$ & $\begin{array}{l}\mathrm{u}=-0.000 \mathrm{p}=1^{\mathrm{b}} \\
(\downarrow 8 \%) \\
\mathrm{u}=-0.122 \mathrm{p}=0.902^{\mathrm{b}} \\
(\downarrow 7 \%)\end{array}$ & $\begin{array}{l}\mathrm{z}=0.152 \mathrm{p}=0.878^{\mathrm{c}} \\
(\uparrow 65 \%) \\
\mathrm{z}=0.152 \mathrm{p}=0.878^{\mathrm{c}} \\
(\uparrow 4 \%)\end{array}$ \\
\hline & $\begin{array}{l}\text { am vs } \\
\mathrm{pm}\end{array}$ & $\begin{array}{l}\mathrm{t}=0.033 \quad \mathrm{p}=0.856^{\mathrm{a}} \\
(\uparrow 6 \%)\end{array}$ & $\begin{array}{l}\mathrm{z}=0.051 \\
\mathrm{p}=0.959^{\mathrm{c}} \\
(\uparrow 7 \%)\end{array}$ & $\begin{array}{l}\mathrm{z}=0.153 \\
\mathrm{p}=0.878^{\mathrm{c}} \\
(\downarrow 32 \%)\end{array}$ & & \\
\hline \multirow[t]{2}{*}{$\begin{array}{l}\text { Total Antioxidant } \\
\text { Status (TAS) } \\
\text { mmol/l }\end{array}$} & $\begin{array}{l}\mathrm{am} \\
\mathrm{pm}\end{array}$ & $\begin{array}{l}0.49 \pm 0.21 \\
0.51 \pm 0.30\end{array}$ & $\begin{array}{l}0.40 \pm 0.15 \\
0.39 \pm 0.17\end{array}$ & $\begin{array}{l}0.52 \pm 0.18 \\
0.47 \pm 0.17\end{array}$ & $\begin{array}{l}\mathrm{t}=-0.979 \mathrm{p}=0.341^{\mathrm{a}} \\
(\downarrow 4 \%) \\
\mathrm{t}=-1.161 \mathrm{p}=0.262^{\mathrm{a}} \\
(\downarrow 23 \%)\end{array}$ & $\begin{array}{l}t=2.323 p=0.144^{a} \\
(\uparrow 30 \%) \\
t=1.137 p=0.300^{a} \\
(\uparrow 20 \%)\end{array}$ \\
\hline & $\begin{array}{l}\text { am vs } \\
\mathrm{pm}\end{array}$ & $\begin{array}{l}\mathrm{t}=0.049 \quad \mathrm{p}=0.826^{\mathrm{a}} \\
(\uparrow 4 \%)\end{array}$ & $\begin{array}{l}\mathrm{t}=0.061 \\
\mathrm{p}=0.806^{\mathrm{a}} \\
(\downarrow 2.5 \%)\end{array}$ & $\begin{array}{l}\mathrm{t}=0.398 \\
\mathrm{p}=0.535^{\mathrm{a}} \\
(\downarrow 10 \%)\end{array}$ & & \\
\hline \multirow[t]{2}{*}{$\begin{array}{l}\text { Total sialic acid } \\
\text { (TSA) } \mathrm{mg} \%\end{array}$} & $\begin{array}{l}\mathrm{am} \\
\mathrm{pm}\end{array}$ & $\begin{array}{l}6.06 \pm 2.10 \\
6.25 \pm 4.51\end{array}$ & $\begin{array}{l}4.78 \pm 1.85 \\
7.19 \pm 3.68\end{array}$ & $\begin{array}{l}5.73 \pm 2.08 \\
3.81 \pm 4.45\end{array}$ & $\begin{array}{l}\mathrm{u}=-1.102 \mathrm{p}=0.270^{\mathrm{b}} \\
(\downarrow 21 \%) \\
\mathrm{u}=0.571 \mathrm{p}=0.567^{\mathrm{b}} \\
(\uparrow 15 \%)\end{array}$ & $\begin{array}{l}\mathrm{t}=1.152 \mathrm{p}=0.297^{\mathrm{a}} \\
(\uparrow 20 \%) \\
\mathrm{t}=0.112 \mathrm{p}=0.741^{\mathrm{a}} \\
(\downarrow 47 \%)\end{array}$ \\
\hline & $\begin{array}{l}\text { am vs } \\
\text { pm }\end{array}$ & $\begin{array}{l}\mathrm{z}=0.533 \quad \mathrm{p}=0.593^{\mathrm{c}} \\
(\uparrow 3 \%)\end{array}$ & $\begin{array}{l}\mathrm{t}=3.424 \\
\mathrm{p}=0.081^{\mathrm{a}} \\
(\uparrow 50 \%)\end{array}$ & $\begin{array}{l}t=1.786 \\
p=0.198^{a} \\
(\downarrow 33 \%)\end{array}$ & & \\
\hline \multirow[t]{2}{*}{$\begin{array}{l}\text { Free sialic acid (FSA) } \\
\mathrm{mg} \%\end{array}$} & $\begin{array}{l}\mathrm{am} \\
\mathrm{pm}\end{array}$ & $\begin{array}{l}2.23 \pm 1.52 \\
2.76 \pm 1.93\end{array}$ & $\begin{array}{l}2.05 \pm 1.11 \\
3.32 \pm 2.57\end{array}$ & $\begin{array}{l}1.94 \pm 1.02 \\
3.22 \pm 3.17\end{array}$ & $\begin{array}{l}t=-0.283 p=0.781^{\mathrm{a}} \\
(\downarrow 8 \%) \\
\mathrm{t}=0.541 \mathrm{p}=0.596^{\mathrm{a}} \\
(\uparrow 20 \%)\end{array}$ & $\begin{array}{l}t=0.043 p=0.836^{\mathrm{a}} \\
(\downarrow 5 \%) \\
\mathrm{t}=0.007 \mathrm{p}=0.934^{\mathrm{a}} \\
(\downarrow 3 \%)\end{array}$ \\
\hline & $\begin{array}{l}\text { am vs } \\
\text { pm }\end{array}$ & $\begin{array}{l}\mathrm{t}=0.146 \quad \mathrm{p}=0.528^{\mathrm{a}} \\
(\uparrow 24 \%)\end{array}$ & $\begin{array}{l}\mathrm{t}=1.885 \\
\mathrm{p}=0.187^{\mathrm{a}} \\
(\uparrow 62 \%)\end{array}$ & $\begin{array}{l}\mathrm{t}=1.316 \\
\mathrm{p}=0.267^{\mathrm{a}} \\
(\uparrow 66 \%)\end{array}$ & & \\
\hline \multirow[t]{2}{*}{$\begin{array}{l}\text { Glycosidically bound } \\
\text { sialic acid (GSA) } \\
\mathrm{mg} \%\end{array}$} & $\begin{array}{l}\mathrm{am} \\
\mathrm{pm}\end{array}$ & $\begin{array}{l}3.82 \pm 1.28 \\
3.50 \pm 2.88\end{array}$ & $\begin{array}{l}3.22 \pm 2.83 \\
3.87 \pm 1.54\end{array}$ & $\begin{array}{l}4.56 \pm 3.93 \\
4.59 \pm 2.30\end{array}$ & $\begin{array}{l}\mathrm{u}=-1.102 \mathrm{p}=0.270^{\mathrm{b}} \\
(\downarrow 16 \%) \\
\mathrm{u}=1.306 \mathrm{p}=0.191^{\mathrm{b}} \\
(\uparrow 11 \%)\end{array}$ & $\begin{array}{l}\mathbf{z}=\mathbf{2 . 7 0 1} \mathbf{p}=\mathbf{0 . 0 0 7}{ }^{\mathbf{c}} \\
(\uparrow \mathbf{4 2 \%}) \\
\mathrm{z}=1.274 \mathrm{p}=0.202^{\mathrm{c}} \\
(\uparrow 17 \%)\end{array}$ \\
\hline & $\begin{array}{l}\mathrm{am} \text { vs } \\
\mathrm{pm}\end{array}$ & $\begin{array}{l}\mathrm{z}=0.770 \quad \mathrm{p}=0.441^{\mathrm{c}} \\
(\downarrow 8 \%)\end{array}$ & $\begin{array}{l}\mathrm{z}=1.172 \\
\mathrm{p}=0.247^{\mathrm{c}} \\
(\uparrow 20 \%)\end{array}$ & $\begin{array}{l}\mathrm{z}=0.533 \\
\mathrm{p}=0.593^{\mathrm{c}} \\
(\uparrow 1 \%)\end{array}$ & & \\
\hline \multirow[t]{2}{*}{$\begin{array}{l}\text { Cortisol (C) } \\
\mu \mathrm{g} / \mathrm{ml}\end{array}$} & $\begin{array}{l}\mathrm{am} \\
\mathrm{pm}\end{array}$ & $\begin{array}{l}18.43 \pm 17.16 \\
7.28 \pm 8.22\end{array}$ & $\begin{array}{l}4.01 \pm 2.74 \\
2.00 \pm 1.21\end{array}$ & $\begin{array}{l}3.48 \pm 2.96 \\
1.24 \pm 0.61\end{array}$ & $\begin{array}{l}\mathrm{u}=-2.653 \mathrm{p}=0.0007^{b} \\
(\downarrow 78 \%) \\
\mathrm{u}=-2.571 \mathrm{p}=0.010^{b} \\
(\downarrow 72 \%)\end{array}$ & $\begin{array}{l}\mathrm{t}=0.171 \mathrm{p}=0.684^{\mathrm{a}} \\
(\downarrow 13 \%) \\
\mathrm{t}=3.055 \mathrm{p}=0.097^{\mathrm{a}} \\
(\downarrow 38 \%)\end{array}$ \\
\hline & $\begin{array}{l}\text { am vs } \\
\text { pm }\end{array}$ & $\begin{array}{l}\mathrm{z}=1.836 \mathrm{p}=0.066^{\mathrm{c}} \\
(\downarrow 60 \%)\end{array}$ & $\begin{array}{l}t=4.523 \\
p=0.047^{a} \\
(\downarrow 50 \%)\end{array}$ & $\begin{array}{l}\mathrm{t}=5.447 \\
\mathrm{p}=0.031^{\mathrm{a}} \\
(\downarrow 64 \%)\end{array}$ & & \\
\hline \multirow[t]{2}{*}{$\begin{array}{l}\text { Calcium }(\mathrm{Ca}) \\
\mathrm{mg} \%\end{array}$} & $\begin{array}{l}\mathrm{am} \\
\mathrm{pm}\end{array}$ & $\begin{array}{l}4.00 \pm 1.47 \\
2.86 \pm 1.60\end{array}$ & $\begin{array}{l}3.92 \pm 1.55 \\
4.40 \pm 1.89\end{array}$ & $\begin{array}{l}5.56 \pm 2.04 \\
5.98 \pm 2.31\end{array}$ & $\begin{array}{l}t=-0.122 p=0.904^{a} \\
(\downarrow 2 \%) \\
t=1.896 p=0.075^{a} \\
(\uparrow 54 \%)\end{array}$ & $\begin{array}{l}\mathrm{t}=4.079 \mathrm{p}=0.058^{\mathrm{a}} \\
(\uparrow 42 \%) \\
\mathrm{t}=2.817 \mathrm{p}=0.110^{\mathrm{a}} \\
(\uparrow 36 \%)\end{array}$ \\
\hline & $\begin{array}{l}\text { am vs } \\
\mathrm{pm}\end{array}$ & $\begin{array}{l}\mathrm{t}=2.488 \quad \mathrm{p}=0.134^{\mathrm{a}} \\
(\downarrow 28.5 \%)\end{array}$ & $\begin{array}{l}\mathrm{t}=0.379 \\
\mathrm{p}=0.545^{\mathrm{a}} \\
(\uparrow 12 \%)\end{array}$ & $\begin{array}{l}\mathrm{t}=0.186 \\
\mathrm{p}=0.671^{\mathrm{a}} \\
(\uparrow 7 \%)\end{array}$ & & \\
\hline \multirow[t]{2}{*}{$\begin{array}{l}\text { Magnesium (Mg) } \\
\text { mg\% }\end{array}$} & $\begin{array}{l}\mathrm{am} \\
\mathrm{pm}\end{array}$ & $\begin{array}{l}0.89 \pm 0.34 \\
0.65 \pm 0.23\end{array}$ & $\begin{array}{l}0.88 \pm 0.33 \\
0.71 \pm 0.27\end{array}$ & $\begin{array}{l}0.94 \pm 0.27 \\
0.71 \pm 0.28\end{array}$ & $\begin{array}{l}\mathrm{u}=-0.204 \mathrm{p}=0.838^{\mathrm{b}} \\
(\downarrow 1 \%) \\
\mathrm{u}=0.367 \mathrm{p}=0.713^{\mathrm{b}} \\
(\uparrow 9 \%)\end{array}$ & $\begin{array}{l}\mathrm{z}=0.866 \mathrm{p}=0.386^{\mathrm{c}} \\
(\uparrow 7 \%) \\
\mathrm{z}=0.051 \mathrm{p}=0.956^{\mathrm{c}} \\
\text { (const) } 0 \%\end{array}$ \\
\hline & $\begin{array}{l}\text { am vs } \\
\mathrm{pm}\end{array}$ & $\begin{array}{l}\mathrm{t}=3.043 \mathrm{p}=0.100^{\mathrm{a}} \\
(\downarrow 27 \%)\end{array}$ & $\begin{array}{l}\mathrm{z}=1.274 \\
\mathrm{p}=0.202^{\mathrm{c}} \\
(\downarrow 81 \%)\end{array}$ & $\begin{array}{l}\mathrm{z}=1.987 \\
p=0.047^{c} \\
(\downarrow 24 \%)\end{array}$ & & \\
\hline
\end{tabular}

a- t-student test; b- U-Mann-Whitney test; c- Wilcoxon test Significant differences at $\mathrm{p}<0.05$

The effect of exercise on relative changes in salivary components concentrations, presented as $100 \%$ baseline value, is illustrated in Fig-1. 


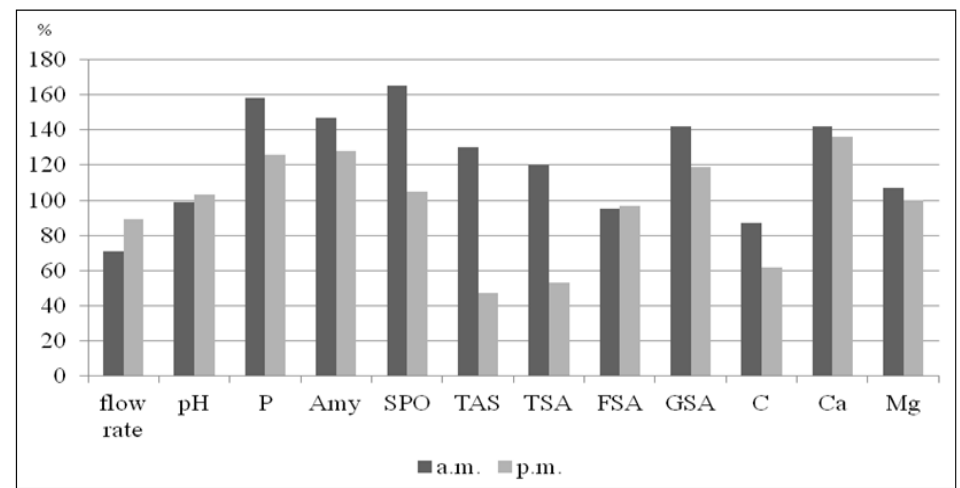

Fig-1: Effect of training on relative changes in salivary parameters, levels before excercise were set at $100 \%$

Salivary components' output in swimmers before training compared with the control group:

In the morning, slightly higher output was noted for all saliva components in swimmers. However, the output was significantly higher only for total protein. The cortisol level output proved to be significantly lower. In the afternoon, slightly higher outputs were observed for all salivary components as well, but they were significantly higher only for total protein and calcium (Fig.2).

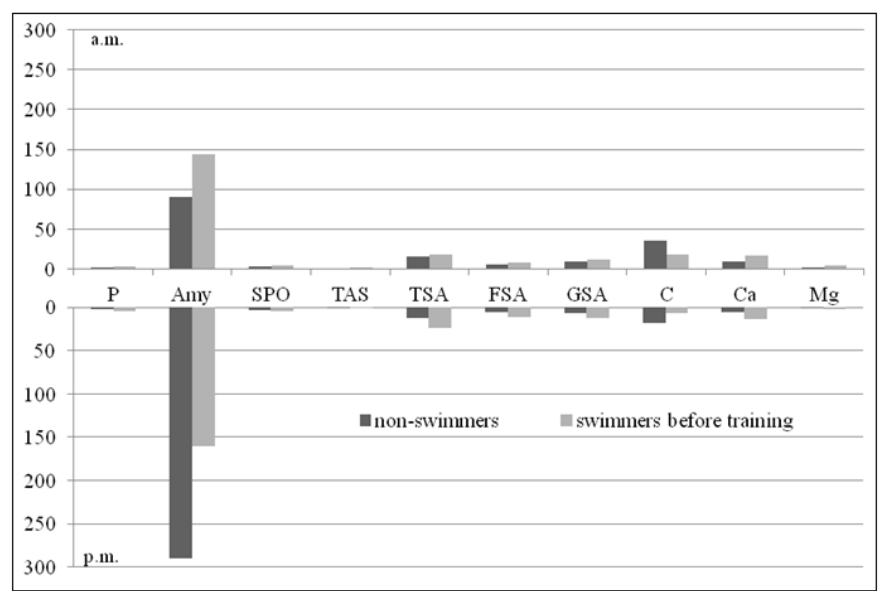

Fig-2: Output of salivary components ( $\mathrm{mg} / \mu \mathrm{g} / \mathrm{U}$ per min.) of swimmers before training and non-swimmers in the morning and afternoon hours

Salivary Components' Output Before and After Training in Swimmers

After the morning training session, a slight increase in the output was observed for all tested salivary components, but it was statistically significant only for total protein, alpha-amylase, calcium, TSA and TAS.
Following the afternoon training session, a slight increase in the output was observed for all salivary components, except for the cortisol for which a declining trend was observed. A significant increase in the output was found for total protein, alpha-amylase, calcium and TAS (Fig-3).

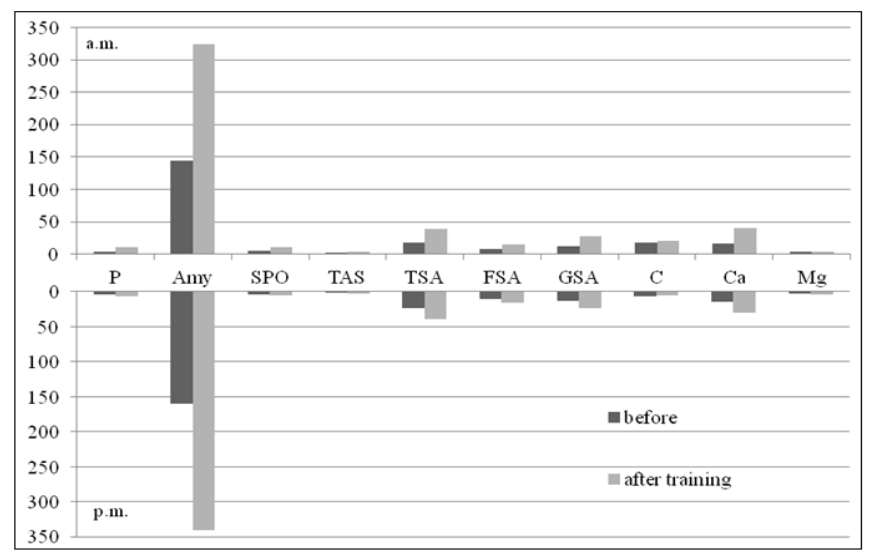

Fig-3: Output of salivary components (mg/ $\mu \mathrm{g} / \mathrm{U}$ per min.) of swimmers before and after training in the morning and afternoon hours. 
Circadian rhythm patterns were demonstrated in the flow rate of saliva and in the levels of the salivary components [17]. This was taken into account while measuring salivary parameters twice a day, i.e. in the morning and in the afternoon. Our data confirms the presence of circadian rhythm patterns in alpha-amylase and cortisol levels contained in saliva. Just like in the Rohlender et al., [18] study, lower alpha-amylase and higher cortisol levels were noted in the control group and in the swimmers' group before training in the morning hours as compared to the afternoon hours. Also, Raff and Trivedi [19] in their study on the circadian rhythm of salivary cortisol showed higher levels of cortisol in the morning hours when compared to the evening hours.

A slight decrease in the salivary flow rate, after swimming exercise observed in our study, is consistent with the previous results $[5,6,20]$. Decrease in the salivary flow rate can be caused by dehydration due to prolonged exercise, which was observed in athletes after a marathon run and other intensive exercise. In our study, swimmers were not exposed to dehydration, because they practiced in the water temperatures ranging from 26 to $28^{\circ} \mathrm{C}$. Therefore excess heat produced by the athlets' own bodies during the exercise was released directly to the environment. Moreover, swimmers were allowed to drink energy drinks at will during the practice. However, Ligtenberg et al., [21] demonstrated a significant increase in the unstimulated salivary flow rate following moderate and intense outdoor running at temperatures ranging between 7 and $15^{\circ} \mathrm{C}$. Dimitriou et al., [5] showed higher salivary flow rates in swimmers in the evening as opposed to the morning, just like in our study, which explains a higher activity of sympathetic nervous system in the morning hours versus at the other times of the day.

During exercises, total protein concentrations in saliva can increase, following an increase of $\beta$ sympathetic activity in salivary glands [3] which was confirmed by our data from both the morning and afternoon training sessions. Our data, just like that of the other studies, showed a significant increase in the total salivary protein levels after physical activity $[1,6$, 21]. De Oliveira et al., [22] suggested that an increase in the total salivary protein levels is caused exclusively by increased alpha-amylase secretion, therefore monitoring of the total protein concentration can serve as an alternative, non-invasive method for determining exercise intensity. Similarly, Diaz et al., [23] considered the assessment of total protein in unstimulated saliva as an attractive marker due to fast testing ability as compared to traditional kinetic or immune assays.

Salivary alpha-amylase is believed to be a biomarker of stress and stimulates sympathetic nervous system activity. It has been successfully applied as an indicator of exercise intensity in well-trained subjects
[24]. The correlation between exercise and alphaamylase activity in saliva has been extensively investigated. However, the obtained data were not consistent, as some studies showed a significant increase, but others showed no such change [25]. In our study a significant increase of salivary alpha-amylase activity after the exercise was observed, regardless of the time of the day. Diaz et al., [23] found an increase of salivary alpha-amylase immediately prior to warming up and 5 minutes after swimming competition in male professional swimmers. Edmonson et al., [26] noticed an increase of the enzyme activity in saliva in elite swimmers with a disability during regular training. Also Sinnott-O'Connor et al., [27] found a significant increase of salivary alpha-amylase activity during intensive training and competition in Paraolympic Swimmers.

Physical exercise is a powerful stimulant provoking the hypothalamic-pituitary axis and cortisol change. It has been assumed that cortisol levels in saliva reflected its concentration in plasma. Physical and psychological stress caused by exercise evokes changes in the cortisol secretion [5, 7, 13]. However, the changes depend on the size and duration of the stimulus. In the case of exercise or training, it is influenced by the type of physical exertion, the impact of training units and time of execution [28]. VanBruggen et al., [29] showed that cortisol responses increased significantly for both serum and saliva only in response to high-intensity exercise on a cycle ergometer. Jacks et al. [30] investigated salivary cortisol levels in response to exercise at 3 different intensities and indicated that only exercise of high intensity and long duration resulted in significant increases in salivary cortisol. Tan and Long [31] noticed that the increase in post-training cortisol in saliva was correlated to the intensity in synchronized swimmers' pool training. O'Connor et al., [32] found a relationship between salivary cortisol after swimming and depressed mood (measured by the Profile of Mood States questionnaire) as they noticed higher salivary cortisol in swimmers compared to the control group during baseline and overtraining, and also a positive correlation with depressed mood during overtraining. A similar result was obtained by Yazdanparastet et al., [33]. Filaire et al., [34] who noticed a significant increase in salivary cortisol concentrations after exercise in the handball players, which did not appear in swimmers. They stated that the type of sport played could influence the concentration of salivary cortisol. Our data did not reveal any significant differences in salivary cortisol levels before and after the swimming practice, either. However, we did observe lower salivary cortisol concentrations in the afternoon as compared to the morning measurements taken before and after exercise, which is in line with the circadian variation [19]. However, in contrast to Dimitrou et al., [5] we did not find elevated levels of salivary cortisol to be caused by the exercise. 
Physical activity leads to an elevated oxygen demand due to an increased rate of metabolism. This is known to lead to an increased production of reactive oxygen species, which are harmful. Therefore, a higher production of antioxidants is a type of biological response deactivation free radials. The salivary antioxidant system consists of various molecules and enzymes such as peroxidase, superoxide dismutase as well as uric acid, ascorbic acid [35]. Sariri et al., [36] and Darmichi et al., [37, 38] found a significant increase in the salivary peroxidase activity, which returned close to the basal value one hour after an exercise, in athletes immediately after an intense run on the treadmill. Total antioxidant status (TAS) concentration was found to be higher in blood plasma of the rugby players after the dosed submaximal physical exercise [39] as well as in saliva of subjects who performed Tai Chi during a period of 6 months [40]. Our data revealed only slightly higher salivary peroxidase and TAS levels following a swimming practice.

Cavas et al., [13] studied activity of selected antioxidative enzymes and free sialic acid concentrations in unstimulated mixed saliva in Judo athletes before and after a two-hour-long training session. His study revealed increased levels of antioxidative enzymes (catalase, superoxide dismutase, glutathione peroxidase) and free sialic acid in saliva after the training, but no changes in the total protein concentration. It was assumed that sialic acid consumes toxic hydrogen peroxide under physiological conditions. It is known that terminal $\mathrm{N}$ acetylneuraminic acid (NANA) residues are removed by neuraminidase enzyme and released particles of sialic acid are recycled, or degraded by other enzymes. Therefore, released $\mathrm{N}$-acetylneuraminic acid represents a form of defense against oxidative destruction, thus enabling us to determine that sialic acid as an alternative marker of oxidative stress. However, our data do not prove such a relationship because no significant increase in the levels of free sialic acid levels has been identified after workouts. Our data have shown a small increase in calcium concentration and a decrease in magnesium content after the swim training. These data are different from Chicharro et al., [14] findings, which have shown a slight decrease of the mean calcium value and a significant increase in magnesium content in the mixed saliva, after a maximal exercise on cycloergometer.

\section{CONCLUSION}

The results of this study suggest a significant increase in the alpha-amylase levels and a tendency for greater total protein concentration in saliva, following an intense swimming exercise, performed in the morning as well as in the afternoon.

\section{REFERENCES}

1. Díaz Gómez MM, Bocanegra Jaramillo OL, Teixeira RR, Espindola FS. Salivary surrogates of plasma nitrite and catecholamines during a 21week training season in swimmers. PLoS One. 2013;8(5):e64043.

2. Nunes LAS, De Macedo DV. Saliva as a diagnostic fluid in sport medicine: potential and limitations. J Bras Med Lab. 2013; 49(5):247-255.

3. Gaviglio CM, Cook, CJ. Relationship between midweek training measures of testosterone and cortisol concentrations and game outcome in professional rugby union matches. The $\mathrm{J}$ of Strength \& Conditioning Res, 2014; 28(12): 34473452.

4. Bardon A, Cedor O, Kollberg H. Cystic fibrosislike changes insaliva of healthy persons subjected to anaerobic exercise. Clin Chim Acta. 1983; 133(3):311-316.

5. Dimitriou L, Sharp NCC, Doherty M. Circadian effects on the acute responses of salivary cortisol and $\operatorname{IgA}$ in well trained swimmers. Br J Sports Med. 2002; 36(4): 260-264.

6. Ljungberg G, Ericson T, Ekblom B, Birkhed D. Saliva and marathon running. Scand J Med Sci Sports. 1997; 7(4):214-219.

7. Walsh NP, Laing SJ, Oliver SJ, Montague JC, Walters R, Bilzon JL. Saliva Parameters as Potential Indices of Hydration Status during Acute Dehydration. Med Sci Sports Exerc. 2004; 36(9):1535-5152.

8. Powell J, DiLeo T, Roberge R, Coca A, Kim JH. Salivary and serum cortisol levels during recovery from intense exercise and prolonged, moderate exercise. Biol. Sport. 2015; 32(2): 91-95.

9. Kanaley JA, Weltman JY, Pieper KS, Weltman A, Hartman ML. Cortisol and Growth hormone resposnses to exercise at different times of day. $\mathbf{J}$ Clin Endocrin Matab. 2001; 86(6):2881-2889.

10. Maruyama Y, Kawano A, Okamoto S, Ando T, Ishitobi Y, Tanaka Y, Inoue A, Imanaga J, Kanehisa M, Higuma H, Ninomiya T. Differences in salivary alpha-amylase and cortisol responsiveness following exposure to electrical stimulation versus the Trier Social Stress Tests. PLoS One. 2012 Jul 30;7(7):e39375.

11. Lowry OH, Rosebrough NJ, Farr AL, Randall RJ. Protein measurement with the Folin phenol reagent. J Biol Chem. 1951; 193(1):265-275.

12. Jenkins RR. Free radical chemistry. Relationship to exercise. Sports Med. 1988; 5(3): 156-170.

13. Cavas L, Arpinar P, Yurdakoc K. Possible interactions between antioxidant enzymes and free sialic acids in saliva: a preliminary study on elite judoists. Int J Sports Med. 2005; 26(10):832-835.

14. Chicharro JL, Serrano V, Ureña R, Gutierrez AM, Carvajal A, Fernandez-Hernando P, Lucia A. Trace elements and electrolytes in human resting mixed saliva after exercise. British journal of sports medicine. 1999 Jun 1;33(3):204-7. 
15. Mansson-Rahemtulla B, Baldone DC, Pruitt KM, Rahemtulla F. Specific assays for peroxidases in human saliva. Arch Oral Biol. 1986; 31(10):661668.

16. Jourdian GW, Dean L, Roseman S. The sialic acids. XI. A periodate-resorcinol method for the quantitative estimation of free sialic acids and their glycosides. J Biol Chem. 1971; 246(2):430-435.

17. Dawes C. Circadian rhythms in human salivary flow rate and composition. J Physiol. 1972; 220(3):529-545.

18. Rohlender N, Nater UM, Wolf JM, Ehlert U, Kirschbaum C. Psychosocial stress-induced activation of salivary alpha-amylase: an indicator of sympathetic activity? Ann N Y Acad Sci. 2004; 1032:258-263.

19. Raff H, Trivedi H. Circadian rhythm of salivary cortisol, plasma cortisol, and plasma ACTH in end-stage renal disease. Endocr Connect. 2013; 2(1):23-31.

20. Bretz WA, Carrilho MR. Salivary parameters of competitive swimmers at gas-chlorinated swimming-pools. J Sports Sci Med. 2013; 12:207208.

21. Ligtenberg AJ, Brand HS, van den Keijbus PA, Veerman EC. The effect of physical exercise on salivary secretion of MUC5B, amylase and lysozyme. Arch Oral Biol. 2015; 60(11):16391644.

22. de Oliveira VN, Bessa A, Lamounier RP, de Santana MG, de Mello MT, Espindola FS. Changes in the salivary biomarkers induced by an effort test. Int J Sports Med. 2010; 31(6):377-381.

23. Diaz MM, Bocanegra OL, Teixeira RR, Soares SS, Espindola FS. Response of salivary markers of autonomic activity to elite competition. Int $\mathbf{J}$ Sports Med. 2012; 33(9):763-8.

24. Edmonds R, Leicht A, Burkett B, McKean M: Cardiac automic and salivary responses to a repeated training bout in elite swimmer. Sports. 2016; 4(1),13-27.

25. Koibuchi E, Suzuki Y. Exercise upregulates salivary amylase in humans (Review). Exp Ther Med. 2014; 7(4):773-777.

26. Edmonds R, Burkett B, Leicht A, McKean M. Effect of chronic training on heart rate variability, salivary $\operatorname{IgA}$ and salivary alpha-amylase in elite swimmers with a disability. PLoS One. 2015;10(6):e0127749.

27. Sinnott-O'Connor C, Comyns T, Nevill AM, Warrington G. Salivary Biomarkers and Training Load during Training and Competition in Paralympic Swimmers. Int. J. Sports Physiol Perform. 2017; 28:1-19.

28. Grzebisz N. the impact of physical effort on cortisol level: a review of the latest report. WSN. 2016;48:206-210.
29. VanBruggen MD, Hackney AC, McMurray RG, Ondrak KS. The relationship between serum and salivary cortisol levels in response to different intensities of exercise. Int $\mathbf{J}$ Sports Physiol Perform. 2011; 6(3):396-407.

30. Jacks DE, Sowash J, Anning J, McGloughlin T, Andres F. Effect of exercise at three exercise intensities on salivary cortisol. J Strength Cond. Res. 2002; 16(2):286-289.

31. Tan AYW, Long Y. Relationships among salivary cortisol, RPE and training intensity in duet synchronised swimmers during pool session training. Br J Sports Med. 2010; 44(14):13-15.

32. O'Connor PJ, Morgan WP, Raglin JS, Barksdale CM, Kalin NH. Mood state and salivary cortisol levels following overtraining in female swimmers. Psychoneuroendocrinology. 1989; 14(4):303-310.

33. Yazdanparast B, Azarbyjani MA, Rasaee MJ, Jourkesh M, Ostojić SM. The effect of different intensity of exercise on salivary steroids concentration in elite female swimmers. Facta Universitatis-series: Physical Education and Sport. 2009; 7(1):69-77.

34. Filaire E, Duché P, Lac G, Robert A. Saliva cortisol, physical exercise and training: influences of swimming and handball on cortisol concentrations in women. Eur J Appl Physiol Occup Physiol. 1996; 74(3):274-278.

35. Liu J, Duan Y: Saliva: a potential media for disease diagnostics and monitoring. Oral Oncol. 2012; 48(7):569-577.

36. Sariri R, Damirchi A, Nazari Y. Salivary antioxidant variations in athletes after intense exercise. Medicina Sportiva. 2013; 9(1):20432050.

37. Damirchi A, Kiani M, Jafarian V, Sariri R. Response of salivary peroxidase to exercise intensity. Eur J Appl Physiol. 2010; 108(6):12331237.

38. Damirchi A, Saati Zareei A, Sariri R. Salivary antioxidants of male athletes after aerobic exercise and garlic supplementation on: A randomized, double blind, placebo-controlled study. J Oral Biol Craniofac Res. 2015; 5(3):146-152.

39. Mrowicka M, Bortnik K, Malinowska K, Kedziora J, Mrowicki J. Total antioxidant status concentration in blood plasma of professional sportsmen after dosed physical exercise. Pol Merkur Lekarski. 2009; 27(157):22-25.

40. Mendoza-Núñez VM, Hernández-Monjaraz B, Santiago-Osorio E, Betancourt-Rule JM, RuizRamos M. Tai Chi exercise increases SOD activity and total antioxidant status in saliva and is linked to an improvement of periodontal disease in the elderly. Oxid Med Cell Longev. 2014;2014:603853. 